\title{
Headache in the development of neurosciences
}

Elio Agostoni ${ }^{1}$

Published online: 12 November 2020

(C) Fondazione Società Italiana di Neurologia 2020

Headache is the most frequent symptom observed in neurological disorders.

Headache is both a symptom and a disease in its primary forms, a warning sign of most secondary forms, and sometimes the only sign of disease for extended periods of time in some acute neurological disorders, such as subarachnoid haemorrhage, in sub-acute ones, such as venous brain thrombosis and CSF hypotension, as well as in chronic neurological disorders.

Among primary headaches, migraine is enjoying great clinical-scientific momentum, enhanced by the discovery of a true and effective prophylactic therapy. Monoclonal antiCGRP antibodies were studied in several trials that provided clinicians with a new therapeutic option to treat patients with migraine and restore a satisfactory quality of life for them. These considerations were translated into practice after the efficacy and safety of monoclonal antibodies in the real world, outside the clinical trial context, were demonstrated. International regulating bodies addressed the issue of the clinical relevance, economic impact and sustainability of this therapeutic option, and defined a few admission and treatment monitoring indicators. Clinicians, scientific societies and the institutions still have to work hard to ensure the best possible treatment for all the entitled parties. The broad domain of secondary headaches, which pose significant differential diagnosis problems and organizational and management challenges, sees the onset of new nosographic entities capable to challenge neurological knowledge and clinical practice. One form of headache now in the limelight is related to a variety of intra-arterial neurovascular procedures. Neurovascular brain procedures are implemented in a growing number of clinical centres, particularly in the light of the evidence of the efficacy

Elio Agostoni

elioclemente.agostoni@ospedaleniguarda.it

1 Neuroscience Department and Niguarda Neuro-Center and Neurology C.S. and Stroke Unit, Grande Ospedale Metropolitano Niguarda, Milan, Italy of revascularization therapies, such as mechanical thrombectomy combined with systemic pharmacological thrombolysis in acute stroke. Ongoing technological development promotes advances in diagnostics and therapy in this field. During or following a procedure, patients may report a headache of variable degrees of severity, which can be either benign or a sign of a severe complication. The International Classification of Headache Disorders (ICHD-3) recognizes different subtypes of headache attributed to endovascular brain procedures, which are included in Chapter 6 (headache attributed to cranial and/or cervical vascular disorders).

The fundamental feature of post-procedure headache is the time relation between the onset of a new headache or the worsening of a pre-existing one and the timing of the procedure. The pathogenic mechanisms considered include the mechanical stimulation of the arterial wall, capable to activate the trigeminal-vascular system, the chemical reaction due to the materials in use, the injected contrast media or the embolic material derived from the mechanical manipulation performed by the endovascular microcatheter. All these conditions may cause inflammatory modifications that are responsible for the onset of headache [1]. Headache can also be a sign of procedural complications, including artery perforation or dissection. Pain is not specific and is typically ipsilateral in extracranial procedures and widespread in intracranial procedures. An important consideration in the management of headache correlated with neurovascular procedures concerns its impact on the patient's clinical-instrumental monitoring. Based on the above, clinicians are quite intuitively concerned about the possible complications of the procedures.

These patients demand a high amount of energy from the neurologist, challenged with the clinical diagnosis and the differential clinical-instrumental diagnosis. Four more aspects are important in the management of these patients: the risk connected with the execution of multiple neuroimaging exams, delayed discharge, patient and healthcare staff anxiety and therapy management. With respect to the latter, scientific literature reports few experiences, and clinicians are therefore bound to make empirical choices about how to treat a 
headache. Inflammation-related pain-generating mechanisms surely deserve some consideration. In clinical practice, steroids are perceived as having some clinical efficacy, and this is also part of my personal and empirical experience: patients improve to the headache-free state if treated, while headache recurs if steroid treatment is discontinued.

This argument deserves some attention from clinical neurosciences and the design of clinical studies dealing with epidemiological issues, pathogenic mechanisms and therapy.

A significant issue that deserves the attention of neurologists is the impact of headache on the general activity of emergency departments in hospitals. Headache is the second main neurological cause for patient access to the ER [2]. In this setting, appropriate identification of the majority of benign primary headaches is a significant challenge, because a misdiagnosed secondary headache can have severe consequences, including permanent neurological deficits and death. Neurologists engaged in emergency settings should have an in-depth knowledge of the whole spectrum of headaches, the warning signs that lead to suspect a secondary headache and the clinical and instrumental diagnostic workup [3].

In my opinion - which is the opinion of a clinician working in a large treatment and research hospital-focus should be made on this important part of neurology at a historical time that is characterized by significant scientific progress, technological innovation and therapeutic opportunities with respect to both primary and secondary headaches. In recent neuroscience history, the development of technologies, particularly for neuroimaging, allowed the profitable application of our anatomo-clinical, semeiotic and pathology knowledge to patients. The improvement of neurosciences today is closely connected with the opportunity to transfer this abundant knowledge to daily practice and to identify and leverage on innovative, efficient and ergonomic organizational models. Interactions between scientific knowledge and organizational algorithms are the true challenge for neurosciences.

\section{Compliance with ethical standards}

Conflict of interest The author declares no conflict of interest.

Ethical approval This article does not contain any study with human subjects.

\section{References}

1. Baron EP, Moskowits SI, Tepper SJ et al (2011) Headache following intracranial neuroendovascular procedures. Headache: The Journal of Head and Face Pain 52:739-748

2. de Falco FA, Sterzi R, Toso V, Consoli D, Guidetti D, Provinciali L, Leone MA, Beghi E (2008) The neurologist in the emergency department. An Italian nationwide epidemiological survey. Neurol Sci 29:67-75

3. Edlow JA, Panagos PD, Godwin SA, Thomas TL, Decker WW, American College of Emergency Physicians (2008) American College of Emergency Physicians. Clinical policy: critical issues in the evaluation and management of adult patients presenting to the emergency department with acute headache. Ann Emerg Med 52(04):407-436

Publisher's note Springer Nature remains neutral with regard to jurisdictional claims in published maps and institutional affiliations. 